\title{
Is the appearance of periarticular new bone formation related to local neurological disability?
}

\author{
A Catz MD, D Snir MD, Z Groswasser MD, L Mendelson MD, P Solzi MD \\ Loewenstein Rehabilitation Hospital, 278 Achuza Street, PO Box 3, Ra'anana 43100, \\ Israel and Sackletr Faculty of Medicine, Tel-Aviv University, Tel-Aviv, Israel.
}

The present work examined the relationship between the appearance of periarticular new bone formation (PNBF) and the presence of local sensorimotor disability, and the relationship between PNBF and the severity of the motor disability. The study population consisted of 18 patients with spinal cord lesions and 18 patients with traumatic brain injury.

The confinement of PNBF below the level of neurological deficit in patients with spinal cord lesions, and mainly to paralysed or paretic limbs in brain injured patients, indicates a possible causal relationship between the presence of sensorimotor disability and PNBF. On the other hand, the high incidence of bilateral PNBF in patients with incomplete spinal lesions and the appearance of PNBF in some nonplegic and even paretic limbs in the brain injured patients, demonstrates the lack of connection between the severity of the motor deficit and the risk of PNBF. It is suggested that local factors which are related to sensorimotor disability are probably involved in PNBF induction, but additional elements may also play a role in the induction of PNBF and in its propagation.

Key words: local factors; sensorimotor disability; periarticular new bone formation; spinal cord lesions; traumatic brain injury.

\section{Introduction}

Periarticular new bone formation (PNBF) is a well known complication of both spinal and craniocerebral lesions. PNBF is related to the neurological deficit, but its underlying pathophysiology is unclear, and its cause is unknown. ${ }^{1,2}$

Both central and local mechanisms have been suggested as possibly responsible for the occurrence of PNBF. Central mechanisms may be genetic in origin, ${ }^{3-5}$ hormonal $^{6}$ or metabolic. ${ }^{7}$ Possible local causes are trauma (including iatrogenic damage during physiotherapy), immobilisation, infection, pressure sores, vasomotor anomalies in the form of bone arteriovenous shunts with vascular hyperplasia in the area of the joint and hypoxia or oedema due to circulatory stasis. $^{8-10}$

The aim of the present study was to evaluate the relative contribution of local disability factors to PNBF in patients with spinal cord lesions and in patients with traumatic brain injury. The study was based on the following hypotheses:

1 The appearance of PNBF depends on the presence of a neurological disability of the affected limb.

2 The appearance of PNBF depends on the severity of the motor disability of the affected limb.

Verification of these hypotheses would imply that local factors that rely on the neurological integrity of the limb have a major role in the induction and propagation of PNBF.

The relationship of the functional impairment caused by PNBF and the motor disability was also investigated.

\section{Patients and methods}

The records of 404 patients with spinal cord lesions and about 3000 with traumatic brain 
injury admitted to the Loewenstein Rehabilitation Hospital between 1965 and 1989 were retrospectively screened. The total yield of cases with findings compatible with a diagnosis of PNBF consisted of 18 spinal and 111 brain injured. Data of the 18 patients with spinal cord lesions (15 males, 3 females) and 18 brain injured patients (11 males, 7 females) whose names were the ones first to be picked up blindly from the shuffled list of 111, were evaluated for neurological disability, PNBF distribution and its functional effect.

Patient age was similar in the 2 groups, ranging from 17 to 65 years (mean, 28.6 years) in the patients with spinal lesions and from 15 to 56 years (mean, 26.9 years) in the brain injured patients.

All brain lesions were traumatic. Of the 18 spinal lesions, 13 were caused by trauma and 4 had various other causes (spinal meningiomatosis, B12 deficiency, multiple aneurysms and multiple sclerosis). The cause of one spinal cord lesion is unknown. Patients with brain injury had been in coma or in a prolonged state of unawareness for periods ranging from 7 to 210 days.

A total of 29 affected joints in the 18 spinal lesion patients and 37 joints in the brain injured patients were examined. The diagnosis was based on $x$-ray findings in all cases. The $x$-ray films were obtained from routine radiographic survey of the large joints (shoulders, elbows, hips, knees) in the brain injured patients. In the patients with spinal cord lesions joints were $\mathrm{x}$-rayed only when clinical findings like pain, swelling or restriction of movement appeared. PNBF was diagnosed when a diffuse cloud-like radio-opacity appeared in the soft tissue near a joint. Frequently, the radio-opaque area became more dense and got a bony appearance, sometimes with fine trabeculae. The x-ray films could show an apparent fusion of the new bone with the pre-existing bones, either in the form of a mass extending from bony prominences like the trochanters, or in the form of a bony bridge between the bones comprising the joints. Time from injury to diagnosis ranged from 45 to 330 days (mean, 123 days) in the first group and from 35 to 205 days (mean, 83 days) in the second group.
Location of the spinal lesions varied: 11 were lower thoracic (T7-T12), 4 were cervical (C5-C8), 2 were lumbar (L1), and one was upper thoracic (T5). All the spinal lesions included sensory and motor components. Eleven patients had an upper motor neuron type lesion, 4 had a lower motor neuron type lesion, and 3 had a mixed type lesion. Thirteen lesions were complete and 5 were incomplete. Of the 18 brain injured patients, 6 had significant sensory deficits with distributions similar to those of the motor impairment; 10 had bilateral motor deficits (tetraparesis), 7 had unilateral motor deficit (4 hemiplegia, 3 hemiparesis), and one had no motor deficit.

Sacral or gluteal pressure sores appeared in 10 patients with spinal lesions ( 9 of them complete) and in 2 brain injured patients. However, the sores were in direct viscinity with PNBF in 4 spinal patients only and in none of the brain injured. Anticoagulant administration was recorded in one case with spinal lesion and in none of the brain injured patients.

The relationship between the appearance of PNBF and the presence of a sensorimotor disability was evaluated by comparing their distributions: upper or lower limbs; unilateral or bilateral. The relationship between the appearance of PNBF and the severity of the motor disability was evaluated by checking the incidence of bilateral PNBF in patients with complete or motor-incomplete bilateral spinal lesions and the presence of PNBF in plegic and in nonplegic limbs.

The analysis of the data was mainly of a qualitative nature due to the rather small number of patients in the groups; statistical significance, however, was calculated, by the Exact Fisher test.

\section{Results}

Evaluating the relationship between the appearance of PNBF and the presence of motor disability, we found that PNBF always appeared below the level of neurological deficit and in plegic or paretic rather than in nonparetic limbs. In 14 of the 18 patients with spinal cord lesions, the neurological deficit was at the thoracic or lumbar level, with the upper limbs free of motor 
disability. In all cases with spinal lesions, PNBF was confined to the lower limbs. Twenty-eight of the 29 affected joints of this group $(97 \%)$ were hips; there was one affected knee joint (3\%) (Table I). In contrast, 17 of the 18 patients with traumatic brain injury suffered neurological impairment in both upper and lower limbs. Accordingly, PNBF appeared in joints of both the upper and lower limbs in this group. Ten of the 17 patients had PNBF in both upper and lower limbs. Of the 37 affected joints, $14(38 \%)$ were elbows, $10(27 \%)$ were shoulders, 11 (29\%) were hips, and 2 (5\%) were knees (Table I). The difference between the patients with spinal lesions and traumatic brain injury in regard to the proportional distribution of PNBF between the upper and the lower limbs, was highly significant $(p<0.001)$. In the group of traumatic brain injuries, bilateral PNBF was noted in 7 of 10 patients with bilateral motor deficit $(70 \%)$ and in 2 of 7 patients with unilateral disability (29\%). Although these proportions did not reach a statistical significance $(p>0.05)$, it seems that bilateral PNBF was more frequent with bilateral motor deficit. PNBF never appeared in a nonparetic limb without affecting the parallel paretic limb of the same patient (Table II).

Evaluating the relationship between the appearance of PNBF and the severity of motor disability, we found that PNBF appeared in limbs that were not completely paralysed, at least as frequently as in limbs with complete plegia. Bilateral PNBF appeared in 4 of the 5 patients $(80 \%)$ with incomplete spinal cord lesions, but in only 7 of the 13 patients $(54 \%)$ with complete lesions (Table II) $(p>0.2)$. In 19 patients (5 with spinal lesions and 14 with brain injury), PNBF developed in limbs that were not completely plegic, including 3 limbs with no residual motor disability. Only in 17 patients (13 with spinal lesions and 4 with brain injury), PNBF developed in completely plegic limbs.

The functional motor state was graded from 1 to 4 (Table III). In the patients with spinal lesions, PNBF did not result in prominent additional functional impairment, regardless of the completeness of the neurological deficit. On the other hand, in 4 brain injured patients, PNBF caused marked functional impairment in either plegic or paretic limbs.

\section{Discussion}

There appears to be a general tendency in the literature to seek a single aetiological factor for disease. Accordingly, a variety of unrelated mechanisms have been proposed as underlying causative elements in PNBF. Sazbon et $a l^{6}$ demonstrated a significant abnormal increase in serum growth hormone concentration after nonspecific stimulation with thyrotropin-releasing factor in brain injured patients with PNBF. A genetic mechanism was proposed by Larson et $a^{4}$ who found a significant increase in the prevalence of HLA-B27 in spinal lesion patients with ectopic ossification. This result, however, was not corroborated by other studies. ${ }^{3,5}$

Other authors have suggested local factors, such as infection, pressure sores, iatrogenic trauma or circulatory stasis and

Table I Distribution of affected joints

\begin{tabular}{llccccc}
\hline & & Hip & Knee & Elbow & Shoulder & Total \\
\hline \multirow{2}{*}{ SCL } & Bilateral & $11 \times 2=22$ & - & - & - & 22 \\
& Unilateral & 6 & 1 & - & - & 7 \\
TBI & Bilateral & $1 \times 2=2$ & $1 \times 2=2$ & $5 \times 2=10$ & $1 \times 2=2$ & 16 \\
& Unilateral & 9 & - & 4 & 8 & 21 \\
\hline
\end{tabular}

$\mathrm{SCL}=$ spinal cord lesions; $\mathrm{TBI}=$ traumatic brain injury. 
Table II Relation of PNBF to presence and to severity of paralysis

\begin{tabular}{llcccc} 
& & & $\begin{array}{c}\text { Unilateral } \\
\text { PNBF in a limb } \\
\text { with motor } \\
\text { deficit }\end{array}$ & Bilateral PNBF & $\begin{array}{c}\text { Unilateral } \\
\text { PNBF only in a } \\
\text { limb without } \\
\text { motor deficit }\end{array}$ \\
\hline \multirow{3}{*}{ SCL } & $\begin{array}{l}\text { Complete } \\
\text { lesion }\end{array}$ & 13 & 6 & 7 & - \\
& $\begin{array}{l}\text { Incomplete } \\
\text { lesion }\end{array}$ & 5 & 1 & 4 & - \\
ThI & Tetraparesis & 10 & 3 & 7 & - \\
& Hemiplegia & 4 & 3 & 1 & 0 \\
& Hemiparesis & 3 & 2 & 1 & - \\
\hline
\end{tabular}

$\mathrm{SCL}=$ spinal cord lesions; $\mathrm{TBI}=$ traumatic brain injury.

oedema. ${ }^{8-11}$ These factors are frequently related to the reduction of movement or sensation in the affected limb. Therefore, if the local factors play a major role in PNBF, then the appearance of PNBF should be related to the presence of a sensorimotor disability in the limb and to the severity of such disability. In addition, a severe motor deficit would be expected to cause a large amount of bone formation with subsequent marked functional impairment.

The importance of local factors in the induction of PNBF is supported by other publications noting the absence of PNBF above the level of neurological deficit ${ }^{12}$ and variable joint involvement in brain injured patients. $^{2}$ The observation that PNBF occurred more frequently in complete rather than in incomplete spinal lesions ${ }^{12}$ led us to believe that the severity of the motor deficit affects the disposition to PNBF.

Our findings support a relationship between the presence of a sensorimotor disability and the appearance of PNBF. Differentiation between the effects of motor and sensory deficits on PNBF was impossible as their distributions were similar. In the patients with spinal cord lesions, PNBF occurred in the lower limbs, below the level of neurological deficit only. In the patients with traumatic brain injury, PNBF appeared also in the upper limbs, usually in those paretic or paralysed. Moreover, patients with bilateral motor disability frequently had bilateral PNBF.

Contrary to our earlier assumption, however, the induction of PNBF is apparently not related to the severity of the motor deficit. The frequency of bilateral PNBF with complete lesions was not significantly

Table III Functional impairment caused by PNBF

\begin{tabular}{lcc}
\hline Grade & SCL & TBI \\
\hline 1 & 14 & 9 \\
2 & 0 & 3 \\
3 & 4 & 2 \\
4 & 0 & 4 \\
\hline
\end{tabular}

Grade 1: Limited limb movements due to PNBF without significant functional impairment.

Grade 2: Limited limb movements not due to PNBF without significant functional impairment.

Grade 3: Limited limb movements not due to PNBF with significant functional impairment.

Grade 4: Limited limb movements due to PNBF with significant functional impairment.

$\mathrm{SCL}=$ spinal cord lesions; $\mathrm{TBI}=$ traumatic brain injury. 
different from that with incomplete lesions, and PNBF did not further impair functional activity, regardless of the completeness of the lesion. Similar findings were presented by Hernandez et al, ${ }^{12}$ who found further functional impairment due to PNBF in only $1 \%$ of patients. In the brain injured patients, PNBF was found not only in the paralysed limbs, but also in limbs with no motor deficit (although these limbs had been temporarily motionless when the patients were in coma). Four patients in this group suffered from a significant functional impairment due to PNBF; however, this impairment appeared with variable motor deficits.

The nature of the PNBF of our patients could be questioned by those who would not consider the PNBF related to pressure sores as 'true bone'. ${ }^{8}$ However, like Lal we consider that pressure sores may not be a direct cause of PNBF, but rather an accompanying phenomenon which is frequently related to complete lesions. ${ }^{11}$ In accordance with this hypothesis, in this study, 9 of the 10 spinal lesions related to pressure sores were complete and only 4 of the sores were in direct viscinity with PNBF. Therefore, patients with pressure sores were not excluded from this study. Another possible concern about the nature of PNBF could be the relation of ossification to bleeding secondary to anticoagulant administration. This consideration can be ignored, however, as only one of the 36 patients was anticoagulated.

The relationship between the presence of a sensorimotor disability and the appearance of PNBF supports the concept that local factors related to the neurological deficit may be involved in PNBF induction. On the other hand, the absence of a link between the severity of the motor disability and the appearance of PNBF or its functional significance, hints that some additional factors play a role in the induction and in the propagation of PNBF.

It seems that the search for multiple risk factors $^{11}$ will eventually lead to a better understanding of the causative mechanisms involved in PNBF and hopefully to its prevention and treatment.

\section{References}

1 Mendelson L, Groswasser Z, Najenson T, Sandbank U, Solzi P (1975) Periarticular new bone formation in patients suffering from severe head injuries. Scand J Rehabil Med 7: 141-145.

2 Garland DE (1988) Clinical observations on fractures and heterotopic ossification in the spinal cord and traumatic brain injured populations. Clin Orthop Related Res 223: 86-101.

3 Weiss S, Groswasser Z, Ohri A, Mizrachi Y, Orgad S, Efter T, Gazit E (1979) Histocompatibility (HLA) antigens in heterotopic ossification associated with neurological injury. J Rheumatol 6(1): 88-91.

4 Larson JM, Michalski JP, Collacott EA, Eltorai D, McCombs CC, Mandorsky JB (1981) Increased prevalence of HLA-B27 in patients with ectopic ossification following traumatic spinal cord injury. Rheumatol Rehabil 20(4): 193-197.

5 Garland DE, Alday B, Venos KG (1984) Heterotopic ossification and HLA antigens. Arch Phys Med Rehabil 65(9): 531-532.

6 Sazbon L, Sack J, Najenson T, Lunenfeld B (1982) Growth hormone and periarticular new bone formation. A causal relationship? A preliminary communication. Scand J Rehabil Med 15: 43-46.

7 Kewalramani LS (1977) Ectopic ossification. Am J Phys Med 56: 99-121.

8 Silver JR (1969) Heterotopic ossification. A clinical study of its possible relationship to trauma. Paraplegia 7: 220-230.

9 Stover SL, Hataway CJ, Zeiger HE (1975) Heterotopic ossification in spinal cord injured patients. Arch Phys Med Rehabil 56: 199-204.

10 Chantraine A, Minaire P (1981) Para-osteo arthropathies, a new theory and mode of treatment. Scand J Rehabil Med 131: 31-37.

11 Lal S, Hamilton BB, Heinemann A, Betts HB (1989) Risk factors for heterotopic ossification in spinal cord injury. Arch Phys Med Rehabil 70: 387-390.

12 Hernandez AM, Forner JV, De la Fuente T, Gonzalez C, Miro R (1978) The para articular ossifications in our paraplegics and tetraplegics: A survey of 704 patients. Paraplegia 16: 272-275. 